\title{
Review: long-term $\beta$-blocker use reduces mortality after myocardial infarction
}

Freemantle $N$, Cleland J, Young P, Mason J, Harrison J. $\beta$ blockade after myocardial infarction: systematic review and meta regression analysis. BMJ 1999 Jun 26;318:1730-7.

QUESTION: In patients with myocardial infarction (MI), do $\beta$-blockers reduce all-cause mortality and recurrent $\mathrm{MI}$ without adverse effects?

\section{Data sources}

Studies were identified by searching databases from their inception to 1997 (Medline, EMBASE/Excerpta Medica, Biosis, Sigle, HealthStar, IHTA, Conference Papers Index, Derwent Drug File, Dissertation Abstracts, International Pharmaceutical Abstracts, Pascal, and Science Citation Index). Bibliographies of relevant studies and reviews were also checked.

\section{Study selection}

Randomised controlled trials were selected if treatment lasted $\geq 1$ day, patients had had an MI, and $\beta$-blockers were compared with placebo or other treatments.

\section{Data extraction}

Data were extracted on patient numbers and characteristics; type, route, and dose of treatments; duration of treatment and follow-up; loss to follow-up; blinding; concealment of randomisation; study inclusion and exclusion criteria; and outcomes (deaths, recurrent MI, and withdrawals).

\section{Main results}

82 trials met the inclusion criteria (54 234 patients). Short-term (treatment duration $<6$ wks from onset of pain) studies $(\mathrm{n}=51)$ had an overall mortality rate of $10.5 \%$. In these studies, $\beta$-blockers did not reduce mortality (odds ratio [OR] for random-effects model $0.96,95 \%$ CI 0.85 to 1.08 ); subgroup analyses did not differ in mortality for individual drugs.

Long-term (treatment duration 6 to $48 \mathrm{mo}$ ) studies $(\mathrm{n}=31)$ had a mortality rate of $9.7 \%$. Mortality was reduced for all $\beta$-blockers combined (OR 0.77, CI 0.69 to 0.85 ) and also for metoprolol (OR 0.80, CI 0.66 to $0.96,7$ studies), propranolol (OR 0.71 , CI 0.59 to $0.77,7$ studies), and timolol (OR 0.59, CI 0.46 to 0.77 , 2 studies). Drugs with cardioselectivity showed no reduction in mortality (OR 1.10, CI 0.89 to 1.39). Drugs with intrinsic sympathomimetic activity showed a trend toward increased mortality (OR 1.19, CI 0.96 to 1.47). Data on re-infarction showed similar trends for a reduction with long-term $\beta$-blocker use. No additional benefit was shown if the initial treatment started with an intravenous dose (OR 0.87, CI 0.61 to 1.22). Subgroup analyses did not differ in reductions in mortality over time. Withdrawal rates ranged from $10 \%$ to $30 \%$ and were similar in the treatment and control groups.

\section{Conclusion}

Long-term treatment (6 to $48 \mathrm{mo}$ ) with $\beta$-blockers reduces mortality in patients who have had a myocardial infarction.

\section{COMMENTARY}

$\beta$-adrenergic receptor blockade in the acute and the convalescent phases of MI is the standard of care ${ }^{1}$ established in a remarkable series of clinical trials. Despite the evidence, $\beta$-blockers after MI continue to be underused. ${ }^{2}$ Freemantle et $a l$, who have collated data from many $\beta$-blocker trials, have shown that, although short-term $\beta$-blocker use does not decrease mortality, long-term $\beta$-blockade trials show consistent benefit. Early intravenous $\beta$-blocker therapy in acute MI is recommended by the joint American College of Cardiology and American Heart Association guidelines for the management of patients with acute MI. ${ }^{1}$ The present review calls into question the benefit of early intravenous use of $\beta$-blockers in acute MI. The widespread use of thrombolysis and primary percutaneous coronary angioplasty (where available) improves prognosis so markedly that any additional short-term benefit of $\beta$-blockers may be hard to prove. Clinicians faced with this question can wonder what role $\beta$-blockers have in treating acute MI. However, the clinical setting often includes an important subset of patients in whom thrombolysis either fails or results in suboptimal outcomes. ${ }^{3}$ Here, $\beta$-blockers are likely to improve mortality, attenuate ischaemic pain, and prevent tachyarrhythmias.

Long-term use of $\beta$-blockers after MI remains unchallenged and is highlighted by the present review. After an MI, patients often develop further clinical and subclinical myocardial damage, which can increase the chance of arrhythmias by altering the heart muscle with more scar tissue. Therefore, $\beta$-blockers should probably be used indefinitely. Should the $\beta$-blockers used be those with selective, non-selective, or intrinsic sympathomimetic activity? This meta-analysis raises interesting but unanswered questions about their relative clinical efficiency. It also seems prudent to use the actual agents that have shown benefit at established doses. The low withdrawal rates with $\beta$-blockers emphasise the tolerability of the treatment.

Kalyanam Shivkumar, MD University of California at Los Angeles Medical Center Los Angeles, California, USA

1 Ryan TJ, Anderson JL, Antman EM, et al. ACC/AHA guidelines for the management of patients with acute myocardial infarction: executive summary. A report of the American College of Cardiology/American Heart Association Task Force on Practice Guidelines (Committee on Management of Acute Myocardial Infarction). Circulation 1996;94:234150.

2 Viskin S, Barron HV. Beta blockers prevent cardiac death following a myocardial infarction: so why are so many infarct survivors discharged without beta blockers? Am J Cardiol 1996;78:821-2.

3 Davies CH, Ormerod OJ. Failed coronary thrombolysis. Lancet 1998;351:1191-6. 